\title{
Nonlinear modelling of a rotating multi-component dusty plasma
}

\author{
I. Kourakis ${ }^{* \dagger}$, U. M. Abdelsalam ${ }^{* *, \ddagger}$, W. M. Moslem ${ }^{\dagger, \S}$ and P. K. Shukla ${ }^{\dagger}$ \\ ${ }^{*}$ Centre for Plasma Physics, Queen's University Belfast, BT7 1 NN Northern Ireland, UK \\ www.kourakis.eu \\ ${ }^{\dagger}$ Ruhr Universität Bochum, Fakultät für Physik und Astronomie, D-44780 Bochum, Germany \\ ${ }^{* *}$ Fakultät für Mathematik, Ruhr-Universität Bochum, D-44780 Bochum, Germany \\ ${ }^{*}$ Department of Mathematics, Faculty of Science, Fayoum University, Egypt

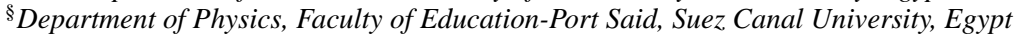

Keywords: Dusty plasma, electrostatic waves, rotating plasma, Zakharov-Kuznetsov equation.

PACS: 52.27.Lw, 52.35.Fp, 52.35.Mw, 52.35.Sb

The Coriolis force may have a dominant role in rotating laboratory plasmas, as well as in space plasma environments, where dusty plasmas (DP) abide [1,2,3]. In this work, we investigate the propagation of nonlinear electrostatic excitations in a four-component DP, consisting of two dust species of opposite polarity $\left(d^{+}\right.$and $d^{-}$, of mass $m$ and charge $\pm Z_{d} e$, respectively), electrons (mass $m_{e}$, charge $e$ ) and ions (mass $m_{i}$, charge $+Z e$ ).

We consider a rotating DP embedded in an external magnetic field $\mathbf{B}=B_{0} \hat{x}$. A twofluid model is employed, for the two dust species (distinguished by indices + and - ). Both Lorentz and Coriolis forces are considered, associated with gyroscopic (Larmor) and mechanical plasma rotation, respectively via the dust Larmor $\omega_{c} \hat{x}= \pm \frac{Z_{d} e B_{0}}{m c} \hat{x}$ and rotation $\Omega_{0} \hat{x}$ frequencies. The density $n_{ \pm}$, velocity $\mathbf{u}_{ \pm}$and potential $\phi$ perturbations vanish at equilibrium. The system of fluid equations

$$
\begin{gathered}
\frac{\partial n_{-,+}}{\partial t}+\nabla \cdot\left(n_{-,+} u_{-,+}\right)=0 \\
m\left(\frac{\partial}{\partial t}+\mathbf{u}_{-} \cdot \nabla\right) \mathbf{u}_{-}=Z_{d} e \nabla \phi-\frac{1}{n_{-}} \nabla p_{-}-\frac{Z_{d} e}{c}\left(\mathbf{u}_{-} \times B_{0} \hat{x}\right)+2 m\left(\mathbf{u}_{-} \times \Omega_{0} \hat{x}\right), \\
m\left(\frac{\partial}{\partial t}+\mathbf{u}_{+} \cdot \nabla\right) \mathbf{u}_{+}=-Z_{d} e \nabla \phi-\frac{1}{n_{+}} \nabla p_{+}+\frac{Z_{d} e}{c}\left(\mathbf{u}_{+} \times B_{0} \hat{x}\right)+2 m\left(\mathbf{u}_{+} \times \Omega_{0} \hat{x}\right) .
\end{gathered}
$$

is closed by the equation(s) of state $p_{\alpha} \sim n_{ \pm}^{5 / 3}$, and by Poisson's equation

$$
\nabla^{2} \phi=4 \pi e\left[Z_{d}\left(n_{-}-n_{+}\right)-Z n_{i}+n_{e}\right] .
$$

We assume that $n_{+, 0} / n_{-, 0} \equiv \delta=1-\gamma$, where $\delta=n_{+, 0} / n_{-, 0}, \gamma=\left(Z_{i} n_{i}-n_{e}\right) / n_{-, 0} \equiv$ $\left.\hat{n}_{0} / n_{-, 0}\right)$, i.e. the $e-i$ background maintains charge neutrality while remaining uniform.

Perturbation theory, introducing slow scales $X=\varepsilon^{1 / 2}(x-\lambda t), Y=\varepsilon^{1 / 2} y$ and $\tau=\varepsilon^{3 / 2}$ $(\varepsilon \ll 1)$ reduces the system $(1)-(4)$ to a ZAKHAROV-KUZNETSOV (ZK) equation

$$
\frac{\partial \phi^{(1)}}{\partial \tau}+A \phi^{(1)} \frac{\partial \phi^{(1)}}{\partial X}+B \frac{\partial^{3} \phi^{(1)}}{\partial X^{3}}+D \frac{\partial^{3} \phi^{(1)}}{\partial X \partial Y^{2}}=0 .
$$

CP1041, Multifacets of Dusty Plasmas - Fifth International Conference on the Physics of Dusty Plasmas edited by J. T. Mendonça, D. P. Resendes, and P. K. Shukla

(C) 2008 American Institute of Physics 978-0-7354-0569-1/08/\$23.00 
where

$$
\begin{gathered}
A=B\left[\frac{3 \delta \lambda^{2}}{\left(\lambda^{2}-2 \delta \sigma_{+}\right)^{3}}-\frac{3 \lambda^{2}}{\left(\lambda^{2}-2\right)^{3}}\right], \\
B=\left[\frac{2 \lambda}{\left(\lambda^{2}-2\right)^{2}}+\frac{2 \delta \lambda}{\left(\lambda^{2}-2 \delta \sigma_{+}\right)^{2}}\right]^{-1}, \\
D=B\left[1+\frac{1}{\Omega_{-}^{2}} \frac{\lambda^{4}}{\left(\lambda^{2}-2\right)^{2}}+\frac{\delta \lambda^{4}}{\Omega_{+}^{2}\left(\lambda^{2}-2 \delta \sigma_{+}\right)^{2}}\right] .
\end{gathered}
$$

We have defined the effective frequencies $\Omega_{ \pm}=\left(2 \Omega_{0} \pm \omega_{c}\right) / \omega_{p,-}$ [where $\omega_{p,-}=$ $\left.\left(4 \pi Z_{d}^{2} e^{2} n_{0} / m\right)^{1 / 2}\right]$ and the positive-to-negative dust temperature ratio $\sigma_{+}=T_{+} / T_{-}$, as well as the (reduced) soliton velocity $\lambda$. Taking $\chi=\ell X+m Y-U \tau$, the solution for the potential $\phi \simeq \varepsilon^{3 / 2} \phi^{(1)}+\mathscr{O}\left(\varepsilon^{2}\right)$ reads

$$
\phi^{(1)}=\phi_{0} \operatorname{sech}^{2}\left(\frac{\chi}{W}\right)
$$

where the amplitude $\phi_{0}=3 U / A \ell$ and the width $W=\sqrt{4 \ell\left(B \ell^{2}+D m^{2}\right) / U}$.

The behavior of these nonlinear solutions is under investigation. Our preliminary results suggest a significant dependence of the width $W$ on the rotation frequency $\Omega_{0}$ and on the dust cyclotron frequency $\omega_{c}$. In particular, an interesting effect is witnessed when $\Omega_{+} \sim 2 \Omega_{0} \pm \omega_{c} \rightarrow 0$, where $W$ diverges, leading to $\phi^{(1)} \rightarrow \phi_{0}$ everywhere; notice the white region in Fig. 1b. Extensions of this work currently underway include the
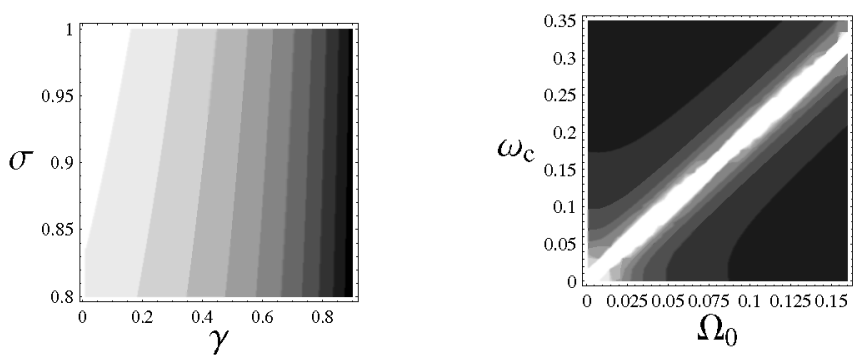

FIGURE 1. Contour plots of: (left frame) the phase velocity $\lambda$ vs. $\sigma$ and $\gamma$ and (right frame) the soliton width $W$ vs. $\Omega_{0}$ and $\omega_{c}$, for $\lambda=0.6, \ell=0.7$ and $\gamma=0.4$. In both plots, higher values are shown as lighter colored areas.

derivation of an extended $\mathrm{ZK}$ equation for critical plasma compositions where $A \simeq 0$, as well as a prediction for the existence of electrostatic double layers in rotating plasmas.

Details on this investigation will be presented in an extensive report, to appear soon.

\section{REFERENCES}

1. F. Verheest, Waves in Dusty Space Plasmas, (Kluwer, Dordrecht, 2000).

2. P. K. Shukla and A. A. Mamun, Introduction to Dusty Plasma Physics, (Institute of Physics, Bristol, 2002).

3. G. C. Das and A. Nag, Phys. Plasmas 13, 082303 (2006).

4. I. Kourakis, U. M. Abdelsalam, W. M. Moslem, P. K. Shukla, in preparation. 